\title{
A note on monotonic power indices, smaller coalitions, and new members
}

\author{
Dominik Karos 1
}

Published online: 12 January 2016

(C) The Author(s) 2016. This article is published with open access at Springerlink.com

\begin{abstract}
Brams' paradox of new members and Shenoy's paradox of smaller coalitions are, in a sense, equivalent. They are both implied by the monotonicity of a power index: while the first is exhibited on every simple game that is not strong, the latter can be observed on every simple game in which players are not almost symmetric. For the Shapley-Shubik index, this symmetry condition is not only necessary but also sufficient to avoid the paradox of smaller coalitions.
\end{abstract}

Keywords Paradox of smaller coalitions · Paradox of new members - Monotonic power indices

\section{Introduction}

The problem of measuring power in simple games appeared to be solved after the discoveries of the Shapley-Shubik index (Shapley 1953; Shapley and Shubik 1954) and the Banzhaf-Coleman index (Banzhaf 1965; Coleman 1971). Of course, these are not the only power measures (see for instance Fara et al 2014, for more on power measures), but they can be considered as the most important ones. However, both indices, when applied to games with different player sets, have properties that are not intuitive. These issues were first described by Brams (1975) and Brams and Affuso (1976) who showed, using the extension of the EU Council in 1973, that adding new players to a game might increase the power of some old players. Brams (1975) called this discovery the paradox of new members.

\footnotetext{
$\otimes$ Dominik Karos

dominik.karos@seh.ax.ac.uk

1 Department of Economics, University of Oxford, St Edmund Hall, Oxford, UK
} 
Shenoy (1979) derived a similar result when applying power indices not only to the grand coalition but to each of its subcoalitions. Intuitively, players should have more power in smaller coalitions. However, it turned out that in some games there are smaller coalitions in which some players are worse off than in the original larger coalition. In this case, a power index would exhibit the paradox of smaller coalitions.

The latter paradox played a crucial role in the theory of coalition formation in simple games. Shenoy (1979) showed that if a power index does not exhibit the paradox of smaller coalitions on a simple game, then there is a minimal winning coalition which is core stable - that is, which will not be left by any group of players. Dimitrov and Haake (2008) relaxed this condition and showed that it is sufficient that any coalition has at least one subcoalition in which each player is not worse off. This seems like a fair generalization of Shenoy's result; however, I will show that in the case of monotonic power indices, it is still quite restricting.

The initial work of Brams and Affuso (1976) derived results on how likely the paradox is to appear. I follow a different approach and investigate the connection between the paradox and the monotonicity of a power index. The relevant definitions can be found in Sect. 2; I also show there that the paradoxes of Shenoy and Brams are, in a sense, equivalent. Two versions of the paradox of new members are investigated in Sect. 3. In particular, it is shown that they are exhibited by any monotonic power index on (almost) every simple game that can be extended. The two versions of the paradox of smaller coalitions are under consideration in Sect. 4, where I show that they are exhibited if players are not almost symmetrical. The condition derived there is both sufficient and necessary for the Shapley-Shubik index. I close the article by briefly discussing the consequences of the incompatibility of monotonicity and the paradoxes' not being exhibited.

\section{Four paradoxes and an axiom}

\subsection{Preliminaries}

Let $\Omega$ be a universe of players, and let $\mathcal{P}_{f}(\Omega)$ be the collection of finite subsets of $\Omega$, called coalitions. ${ }^{1}$ Throughout the paper, when I talk about a player set $N, N$ is finite. A simple game is a function $v: \mathcal{P}_{f}(\Omega) \rightarrow\{0,1\}$ with $v(\emptyset)=0$, such that $v(S) \leq v(T)$ for all $S, T \in \mathcal{P}_{f}$ with $S \subseteq T$, and $v(S)+v(T) \leq 1$ for any two disjoint coalitions $S, T$; the latter property is called properness. If $v(S)=0$ for all coalitions $S, v$ is called the zero game.

A coalition $S$ with $v(S)=1$ is called winning. Let $S$ be winning. If $v(S)-$ $v(S \backslash\{i\})=1$ then $i$ is called pivotal (in $S$ with respect to $v$ ). If $S$ is such that each $i \in S$ is pivotal, $S$ is called minimal winning. The set of minimal winning coalitions is denoted by $\mathcal{M}(v)$. The carrier $c(v)$ of a simple game $v$ is defined as $c(v)=\bigcup_{S \in \mathcal{M}(v)} S$. Throughout the paper, I consider games with finite carrier. If $|\mathcal{M}(v)|=1$, then $v$ is called unanimous. If $v(S)-v(S \backslash\{i\})=1$ for all winning

\footnotetext{
1 The reason why I introduce a universe of players instead of working on a fixed player set $N$ lies in the nature of the problem: I am interested in the effects on power indices if the player set is changed.
} 
coalitions $S$ then $i$ is called a veto player (with respect to $v$ ). If $i \notin c(v)$, then $i$ is called a null player with respect to $v^{2}$ For a coalition $S$, the subgame $v_{S}$ is defined as $v_{S}(T)=v(S \cap T)$ for all coalitions $T$. In particular, $c\left(v_{S}\right) \subseteq S$.

A power index $\varphi$ maps a simple game $v$ and player set $N$ with $c(v) \subseteq N$ into $\mathbb{R}^{N}$. A power index $\varphi$ satisfies the null player axiom if $\varphi_{i}(v, N)=0$ for all $i \notin c(v)$. I will use the null player axiom only for a few remarks, it will not be necessary for the main results. In particular, I will formulate all paradoxes and theorems for arbitrary player sets that include the carrier of the respective game.

\subsection{New members and smaller coalitions}

In Brams (1975), the author derives several fundamental properties, a power index should satisfy. One of them is the property of new members. Let $v$ be a simple game. An extension of $v$ is a simple game $u$ such that $\mathcal{M}(v) \subseteq \mathcal{M}(u)$ and $c(v) \subsetneq c(u)$. A simple game $v$ is extendable if there is an extension $u$ of $v$. Expecting that the addition of new players to a game would not make old players more powerful, Brams (1975) formulated the following paradox.

Strong paradox of new members A power index $\varphi$ exhibits the strong paradox of new members on a simple game $v$ if there is an extension $u$ of $v$ and a player $i \in c(v)$ such that $\varphi_{i}(u, N)>\varphi_{i}(v, N)$ for all player sets $N$ with $c(u) \subseteq N$.

The definition of an extension is quite strict (in Lemma 1, the set of extendable simple games is characterized), and does not capture a very prominent historical case of an extension of a council: namely, the extension of the EU Council of Ministers in 1973 (see Table 1) that has been analyzed by Brams and Affuso (1976). It can easily be seen that some coalitions (for instance, France, Germany and Italy) had a majority before but not after 1973. A weak extension of a simple game $v$ is a simple game $u$ such that $c(v) \subsetneq c(u)$ and $v(S) \geq u_{c(v)}(S)$ for all coalitions $S$. This means that instead of only adding new winning coalitions, old winning coalitions might be removed. Clearly, every non-trivial extension is a weak extension with $v(S)=u_{c(v)}(S)$.

Weak paradox of new members A power index $\varphi$ exhibits the weak paradox of new members on a simple game $v$ if there is a weak extension $u$ of $v$ and a player $i \in c(v)$ such that $\varphi_{i}(u, N)>\varphi_{i}(v, N)$ for all player sets $N$ with $c(u) \subseteq N$.

As every extension of a game is also a weak extension, the paradox of new members indeed implies its weak version. Note that a weak extension of a game can easily be obtained by keeping the votes of old players constant, adding new players with votes, and increasing the quota that is necessary for a coalition to be winning (see, for instance, the extension of the EU Council in 1979 in Table 1).

\footnotetext{
${ }^{2}$ Equivalently, $i$ is a null player if $v(S)=v(S \backslash\{i\})$ for each coalition $S$.
} 
Table 1 Votes and quotas in the EU Council before the Treaty of Nice

\begin{tabular}{|c|c|c|c|c|c|}
\hline Country & $1957^{\mathrm{a}}$ & $1973^{b}$ & $1979^{c}$ & $1985^{\mathrm{d}}$ & $1995^{\mathrm{e}}$ \\
\hline France & 4 & 10 & 10 & 10 & 10 \\
\hline Germany & 4 & 10 & 10 & 10 & 10 \\
\hline Italy & 4 & 10 & 10 & 10 & 10 \\
\hline UK & - & 10 & 10 & 10 & 10 \\
\hline Spain & - & - & - & 8 & 8 \\
\hline Belgium & 2 & 5 & 5 & 5 & 5 \\
\hline The Netherlands & 2 & 5 & 5 & 5 & 5 \\
\hline Greece & - & - & 5 & 5 & 5 \\
\hline Portugal & - & - & - & 5 & 5 \\
\hline Sweden & - & - & - & - & 4 \\
\hline Austria & - & - & - & - & 4 \\
\hline Denmark & - & 3 & 3 & 3 & 3 \\
\hline Ireland & - & 3 & 3 & 3 & 3 \\
\hline Finland & - & - & - & - & 3 \\
\hline Luxembourg & 1 & 2 & 2 & 2 & 2 \\
\hline Total & 17 & 58 & 63 & 76 & 87 \\
\hline Quota & 12 & 41 & 45 & 54 & 62 \\
\hline
\end{tabular}

a Art. 148, Treaty establishing the European Economic Community (EEC Treaty, "Treaty of Rome")

b Art. 8, Council Decision of the European Communities of 1 January 1973 adjusting the documents concerning the accession of new member States to the Euopean Communities

c Art. 14, Act concerning the conditions of accession of the Hellenic Republic and the adjustments to the Treaties

d Art. 14, Act concerning the conditions of accession of the Kingdom of Spain and the Portuguese Republic and the adjustments to the Treaties

e Art. 8, Decision of the Council of the European Union of 1 January 1995 adjusting the instruments concerning the accession of new Member States to the European Union

A similar paradox in a different framework appeared in Shenoy (1979). The author was not interested in the extension of games by new players. Rather, he applied power indices on games and subgames. In the expectation that players would be more powerful in smaller coalitions, he formulated the following paradox.

Weak paradox of smaller coalitions A power index $\varphi$ exhibits the weak paradox of smaller coalitions on a simple game $v$ if there are winning ${ }^{3}$ coalitions $S, T \subseteq c(v), T \subseteq S$, and $i \in c\left(v_{T}\right)$ such that $\varphi_{i}\left(v_{S}, N\right)>\varphi_{i}\left(v_{T}, N\right)$ for all player sets $N$ with $c\left(v_{S}\right) \subseteq N$.

Originally, this paradox was not called weak; however, given more recent developments, this name seems appropriate. Dimitrov and Haake (2008) commented on this paradox as it plays a role when looking for stable coalitions. They introduced a

\footnotetext{
3 If $S$ is not winning then $v_{S}$ is the zero game and power in $v_{S}$ is not very interesting.
} 
weaker condition on a power index and a simple game by requiring that the following stronger paradox is not exhibited.

Strong paradox of smaller coalitions A power index $\varphi$ exhibits the strong paradox of smaller coalitions on a simple game $v$ if there is a winning coalition $S \subseteq c(v)$ such that for all $T \subseteq S$ there is $i \in c\left(v_{T}\right)$ with $\varphi_{i}\left(v_{S}, N\right)>$ $\varphi_{i}\left(v_{T}, N\right)$ for all player sets $N$ with $c\left(v_{S}\right) \subseteq N$.

Hence, if each (non-minimal) winning coalition has at least one winning subcoalition where each member gets at least the same payoff, a power index does not exhibit this stronger paradox. The following easy observation closes this subsection.

Proposition 1 A power index $\varphi$ exhibits the strong paradox of new members on a simple game $v$ if and only if there is a simple game $u$ such that $\varphi$ exhibits the weak paradox of smaller coalitions on $u$.

Proof Let $\varphi$ exhibit the weak paradox of smaller coalitions on the simple game $u$. Then, there are winning coalitions $S, T \subseteq c(u)$ and a player $i \in c\left(u_{T}\right)$ such that $T \subseteq S$ and $\varphi_{i}\left(u_{S}, N\right)>\varphi_{i}\left(u_{T}, N\right)$ for all player sets $N$ with $c\left(u_{S}\right) \subseteq N$. As $u_{S}$ is an extension of $u_{T}$, the strong paradox of new members follows.

On the other hand, if $\varphi$ exhibits the strong paradox of new members on $v$, there are an extension $u$ of $v$ and a player $i \in c(v)$ such that $\varphi_{i}(u, N)>\varphi_{i}(v, N)$ for all player sets $N$ with $c(u) \subseteq N$. As $v=u_{c(v)}$, the weak paradox of smaller coalitions follows.

\subsection{Monotonic power indices}

In the next two sections, I show that the following axiom, which is satisfied for instance by the Shapley-Shubik index (Shapley and Shubik 1954) and the Banzhaf-Coleman index (Banzhaf 1965; Coleman 1971), is responsible for the paradoxes. ${ }^{4}$

Monotonicity A power index $\varphi$ is called monotonic ${ }^{5}$ if for all simple games $u, v$ and all $N$ where $c(u) \cup c(v) \subseteq N$ it holds that $\varphi_{i}(v, N)>\varphi_{i}(u, N)$ for all $i \in N$ such that

$$
\begin{aligned}
& v(S)-v(S \backslash\{i\}) \geq u(S)-u(S \backslash\{i\}) \quad \text { for all } S \subseteq N, \\
& \text { and } \quad v(S)-v(S \backslash\{i\})>u(S)-u(S \backslash\{i\}) \quad \text { for some } S \subseteq N \text {. }
\end{aligned}
$$

It can easily be seen that if $i$ is a null player in $u$ but not in $v$ then monotonicity implies that $i$ has strictly greater power in $v$ than in $u$; in particular, if the null player axiom is, additionally, satisfied then $i$ 's power in $v$ is strictly positive.

\footnotetext{
${ }^{4}$ Kóczy (2009) shows that the null player axiom, together with a normalization, implies the strong paradox of new players for all simple games. This is not true here as null players are excluded from the set of players that can profit from an extension. In that sense, the paradox here is stronger than the one in Kóczy (2009).

5 Note that this definition is the one introduced in Sagonti (1991) and is slightly different from the definition given in Young (1985) to characterize the Shapley value.
} 


\section{The paradox of new members}

Before any results on the exhibition of the strong paradox of new players are derived, I shall answer the question: what games are extendable at all? To formulate the next lemma, I need the following definition. A simple game $v$ is called strong if $v(S)+v(c(v) \backslash S)=1$ for all $S \subseteq c(v)$. That means, it is not only required that the complement of a winning coalition [in $c(v)$ ] is losing, but also that the complement of a losing coalition [in $c(v)]$ is winning.

Lemma 1 A simple game $v$ is extendable if and only if it is not strong.

Proof Let $v$ be a strong game. Then, there are exactly $2^{|c(v)|-1}$ winning coalitions contained in $c(v)$. Suppose there is an extension $u$ of $v$. Then, $v$ has exactly $2^{|c(u)|-1}$ winning coalitions that are contained in $c(u)$. As $u$ is an extension of $v$, it must have at least $2^{|c(u)|-1}+1$ winning coalitions in $c(u)$. Hence, $u$ cannot be proper, i.e., it cannot be an extension of $v$.

On the other hand, let $v$ not be strong. Then, there is a nonempty coalition $S$ such that $v(S)=v(c(v) \backslash S)=0$. Let $k \notin c(v)$ and $u$ be the (unique) simple game with minimal winning coalitions $\mathcal{M}(v) \cup\{S \cup\{k\}\}$. Then, $u$ is a non-trivial extension of $v$.

The good news is that extendable games can easily be identified. From a theoretical perspective, these games can now easily be handled and much is already known about them. The following theorem, however, shows that if a simple game is extendable then any monotonic power index exhibits the strong paradox of new members on it.

Theorem 1 Let $\varphi$ be a monotonic power index. Then, $\varphi$ does not exhibit the strong paradox of new members on a simple game $v$ if and only if $v$ is strong.

Proof If $v$ is strong, it is not extendable and thus $\varphi$ cannot exhibit the paradox. So, let $v$ not be strong. Then, there is a nonempty coalition $S \subseteq c(v)$ such that $v(S)=$ $v(c(v) \backslash S)=0$. Let $k \notin c(v)$ and let $u$ be the simple game with minimal winning coalitions $\mathcal{M}(v) \cup\{S \cup\{k\}\}$. Obviously, $c(u)=c(v) \cup\{k\}$. Let $N$ be a player set containing $c(u)$, let $i \in S$, and let $T \subseteq N$. Suppose that $T$ is such that $i$ is pivotal in $T$ with respect to $v$. As $k \notin c(v)$, this means that $i$ is also pivotal in $T \backslash\{k\}$ with respect to $v$. In particular, $u(T) \geq u(T \backslash\{k\})=v(T \backslash\{k\})=1$. Further, the only minimal winning coalition with respect to $u$ that contains $k$ is $S \cup\{k\}$, and therefore it contains $i$ as well. This means that $k$ cannot be pivotal (with respect to $u$ ) in any coalition that does not contain $i$. Hence, $u(T \backslash\{i\})=u(T \backslash\{i, k\})=v(T \backslash\{i, k\})=0$. Therefore,

$$
u(T)-u(T \backslash\{i\}) \geq v(T)-v(T \backslash\{i\})
$$

for all $T \subseteq N$ in which $i$ is pivotal with respect to $v$. If $T$ is such that $i$ is not pivotal in $T$ with respect to $v$, then $v(T)-v(T \backslash\{i\})=0$ and Eq. (1) holds as well. Further,

$$
u(S \cup\{k\})-u((S \cup\{k\}) \backslash\{i\})=1>0=v(S \cup\{k\})-v((S \cup\{k\}) \backslash\{i\})
$$

as $v(S \cup\{k\})=0$. Hence, by monotonicity, $\varphi_{i}(u, N)>\varphi_{i}(v, N)$. 
The following corollary is an easy consequence of the foregoing proof. It states that for every simple game $v$ that is not strong and for every player, whether or not he is a null player with respect to $v$, an extension of $v$ can be found that makes this player better off.

Corollary 1 Let $\varphi$ be a monotonic power index and let $v$ be a simple game that is not strong. Then, for each $i \in \Omega$, there is an extension $u$ of $v$ such that $\varphi_{i}(u, N)>$ $\varphi_{i}(v, N)$ for each $N$ with $c(u) \cup\{i\} \subseteq N$.

Proof Let $v$ be a simple game that is not strong. If $i \in c(v)$, there is $S \subseteq c(v)$ such that $v(S)=v(c(v) \backslash S)=0$ and $i \in S$. Using the construction in the proof of Theorem 1 , an extension $u$ of $v$ can be obtained with $\varphi_{i}(u, N)>\varphi_{i}(v, N)$ for all $N$ with $c(v) \subseteq N$.

If $i \notin c(v)$, there is $S \subseteq c(v)$ such that $v(S)=v(c(v) \backslash S)=0$. Let $u$ be the simple game with minimal winning coalitions $\{S \cup\{i\}\} \cup \mathcal{M}(v)$. Then, by monotonicity, $\varphi_{i}(u, N)>\varphi_{i}(v, N)$ for each $N$ with $c(u) \cup\{i\} \subseteq N$, as $i$ is not pivotal in any coalition with respect to $v$ but is pivotal in $S \cup\{i\}$ with respect to $u$.

The remainder of this section will deal with weak extensions. Note that every game can be weakly extended; I have already mentioned how it can be done for weighted voting games in Sect. 2. And, again, monotonicity will imply the paradox on almost all games. A dictatorship is a simple game $v$ with $c(v)=\{i\}$ for a player $i \in \Omega$. In such a game, a decision can be made by $i$ only, independently of the player set that $v$ is applied to. Note that if there is a veto player in a strong game $v$ then $v$ is a dictatorship.

Theorem 2 Let $\varphi$ be a monotonic power index. Then, $\varphi$ exhibits the weak paradox of new members on all simple games that are not dictatorships.

Proof Let $v$ be a game that is not a dictatorship and let $N^{\prime}$ be a player set with $c(v) \subseteq N^{\prime}$. I first show that there exist a game $v^{\prime}$ and a player $i \in c\left(v^{\prime}\right)$ such that $v^{\prime}$ is not strong, $v^{\prime}(S) \leq v(S)$ for all coalitions $S$, and $\varphi_{i}\left(v, N^{\prime}\right) \leq \varphi_{i}\left(v^{\prime}, N^{\prime}\right)$. If $v$ is not strong, choose $v^{\prime}=v$. If $v$ is strong, there is $i \in c(v)$ that is not contained in all minimal winning coalitions (otherwise, $v$ would be a dictatorship). Let $S \in \mathcal{M}(v)$ such that $i \notin S$ and let $v^{\prime}$ be the simple game that is defined by

$$
v^{\prime}(T)= \begin{cases}v(T), & \text { if } T \neq S \\ 0, & \text { if } T=S .\end{cases}
$$

Then, $v^{\prime}(T)-v^{\prime}(T \backslash\{i\}) \geq v(T)-v(T \backslash\{i\})$ for all $T \subseteq N^{\prime}$ and

$$
v^{\prime}(S \cup\{i\})-v^{\prime}(S)=v(S \cup\{i\})-0=1>v(S \cup\{i\})-v(S) .
$$

Hence, $\varphi_{i}\left(v, N^{\prime}\right)<\varphi_{i}\left(v^{\prime}, N^{\prime}\right)$ by monotonicity of $\varphi$.

By Corollary 1, there is an extension $u$ of $v^{\prime}$ such that $\varphi_{i}(u, N)>\varphi_{i}\left(v^{\prime}, N\right) \geq$ $\varphi_{i}(v, N)$ for all $N$ with $c(u) \subseteq N$. Since, in particular, $u$ is a weak extension of $v$, the claim is proved.

The following corollary is now clear and the proof is omitted. 
Corollary 2 Let $\varphi$ be a monotonic power index and let $v$ be a simple game which is not a dictatorship. Then, for each $i \in \Omega$, there is a weak extension $u$ of $v$ such that $\varphi_{i}(u, N)>\varphi_{i}(v, N)$ for each $N$ with $c(u) \subseteq N$.

\section{The paradox of smaller coalitions}

The strong paradox of new members and the weak paradox of smaller coalitions are equivalent in the sense that if one is exhibited by a power index on an arbitrary simple game then there is another game on which the other one is exhibited. Nonetheless, they are not exhibited on the same set of games. In the next subsection, a condition on simple games is derived that is necessary for the non-exhibition of the strong paradox of smaller coalitions by a monotonic power index. In Sect. 4.2, I will show that this condition is also sufficient in case of the Shapley-Shubik index.

\subsection{Avoiding the paradox of smaller coalitions}

The key problem of monotonic power indices is that veto players have more power in larger coalitions than in smaller ones. The next lemma illustrates this observation. Recall that $\mathcal{M}(v)$ is the collection of minimal winning coalitions with respect to $v$. For a player $i$, let $\mathcal{M}_{i}(v)$ be the collection of those minimal winning coalitions that contain $i$. Clearly, $\mathcal{M}_{i}(v) \neq \emptyset$ if and only if $i \in c(v)$.

Lemma 2 Let $\varphi$ be a monotonic power index and let $v$ be a simple game such that $\varphi$ does not exhibit the strong paradox of smaller coalitions on $v$. Then, for each winning coalition $S$, either $v_{S}$ is an unanimous game or there is no veto player with respect to $v_{S}$.

Proof Let $S$ be a winning coalition with players $i, j \in S$ such that $i$ is pivotal in $S$ and $j$ is neither null player nor pivotal. Let $N$ be a player set with $c\left(v_{S}\right) \subseteq N$ and let $T \subseteq N$ be such that $v_{S \backslash\{j\}}(T)-v_{S \backslash\{j\}}(T \backslash\{i\})=1$. Then, $v_{S}(T)=1$ and $v_{S}(T \backslash\{i\}) \leq v(S \backslash\{i\})=0$. On the other hand, let $T \subseteq N$ such that $T \in \mathcal{M}_{j}\left(v_{S}\right)$. (Such a $T$ exists as $j$ is not a null player in $S$.) Then, $i$ is pivotal in $T$ with respect to $v_{S}$ but $v_{S \backslash\{j\}}(T)=v_{S}(T \backslash\{j\})=0$. Hence, $\varphi_{i}\left(v_{S}, N\right)>\varphi_{i}\left(v_{S \backslash\{j\}}, N\right)$ by strong monotonicity - that is, $\varphi$ exhibits the strong paradox of smaller coalitions on $v$.

Lemma 2 reveals that a monotonic power index will exhibit the paradox on a number of simple games. This has been elaborated for instance in Karos (2014). The next theorem characterizes the class of simple games that have the property described in Lemma 2.

Theorem 3 Let $v$ be a simple game. Then, $v$ satisfies the necessary condition of Lemma 2 if and only if $\left|\mathcal{M}_{j}\left(v_{S}\right)\right|=\left|\mathcal{M}_{k}\left(v_{S}\right)\right|$ for all winning coalitions $S \subseteq c(v)$ and all $j, k \in c\left(v_{S}\right)$.

Proof First, let $\left|\mathcal{M}_{i}\left(v_{S}\right)\right|=\left|\mathcal{M}_{j}\left(v_{S}\right)\right|$ for all winning coalitions $S$ and all $i, j \in$ $c\left(v_{S}\right)$. Let $T$ be a winning coalition. If $\mathcal{M}_{i}\left(v_{T}\right)=\mathcal{M}\left(v_{T}\right)$ for all $i \in c\left(v_{T}\right)$, then 
$v_{T}$ is unanimous. If $\left|\mathcal{M}_{i}\left(v_{T}\right)\right|<\left|\mathcal{M}\left(v_{T}\right)\right|$ for all $i \in c\left(v_{T}\right)$, then there are no veto players with respect to $v_{T}$. Hence, $v$ satisfies the condition in Lemma 2 .

Now, let $v$ be a simple game such that for each winning coalition $S$, the game $v_{S}$ is either unanimous or does not contain any veto players. The claim is proved by induction on the cardinality of $c(v), n$. For $n=1$ and $n=2$, it is obviously true. So, let $v$ be such that $n=3$. Then, $v$ is either the three player unanimity game or the symmetric game in which each coalition of at least two players is winning. In both cases, the theorem is true.

Let now $n \geq 4$. If $v$ is the unanimous game, the claim is clearly true. So let $v$ not be unanimous. In this case, there cannot be any veto players with respect to $v$ by our initial condition. In particular, $v_{c(v) \backslash\{i\}}$ is not the zero game for any $i \in c(v)$. For a player $i \in c(v)$ let

$$
D_{i}=\left\{l \in c(v) \backslash\left\{i: i \notin c\left(v_{c(v) \backslash\{l\}}\right)\right\}\right.
$$

and

$$
E_{i}=\left\{l \in c(v): i \in c\left(v_{c(v) \backslash\{l\}}\right)\right\} .
$$

In words, $D_{i}$ contains all those players $l$ that "help" $i$ in the sense that after one of them is removed from the game, $i$ becomes a null player. The set $E_{i}$ contains those players $l$ on whose presence $i$ does not depend: If $l$ is removed, $i$ still has some (maybe less) power. Note that $c(v)$ can be partitioned into $D_{i}, E_{i}$ and $\{i\}$. In particular, $E_{i} \neq \varnothing$ as otherwise $c(v) \in \mathcal{M}_{i}(v)$ in contradiction to $v$ 's not being unanimous. I first show that $\left|\mathcal{M}_{j}(v)\right|=\left|\mathcal{M}_{k}(v)\right|$ for all $j, k \in c(v)$. Consider two cases.

1. Let $k \in D_{j}$. In this case $\mathcal{M}_{j}(v) \subseteq \mathcal{M}_{k}(v)$ or, equivalently, $\mathcal{M}\left(v_{c}(v) \backslash\{k\}\right) \subseteq$ $\mathcal{M}\left(v_{c}(v) \backslash\{j\}\right)$. I will show that these two sets are equal. For this purpose, note the following observation. Let $i \in E_{j}$ and $l \in D_{j}$. Then, $\mathcal{M}_{j}\left(v_{c(v) \backslash\{i\}}\right) \subseteq \mathcal{M}_{l}\left(v_{c}(v) \backslash\{i\}\right)$ and the induction hypothesis delivers that $\left|\mathcal{M}_{j}\left(v_{c}(v) \backslash\{i\}\right)\right|=\left|\mathcal{M}_{l}\left(v_{c}(v) \backslash\{i\}\right)\right|$. Hence, $\mathcal{M}_{j}\left(v_{c}(v) \backslash\{i\}\right)=\mathcal{M}_{l}\left(v_{c(v) \backslash\{i\}}\right)$ for all $i \in E_{j}$ and all $l \in D_{j}$.

Assume now that $\mathcal{M}_{j}(v) \subsetneq \mathcal{M}_{k}(v)$-that is, there is a coalition $S \in$ $\mathcal{M}_{k}\left(v_{c(v) \backslash\{j\}}\right)$. Then, $E_{j} \subseteq S$. For, if there were $i \in E_{j} \backslash S$, then $S \in$ $\mathcal{M}_{k}\left(v_{c}(v) \backslash\{i\}\right)=\mathcal{M}_{j}\left(v_{c}(v) \backslash\{i\}\right.$ ) (as seen before), which would mean that $S$ were a minimal winning coalition containing $j$, in contradiction to $S \in \mathcal{M}\left(v_{c}(v) \backslash\{j\}\right)$.

Recall that $E_{j} \neq \emptyset$, let $i \in E_{j}$, and let $T \in \mathcal{M}\left(v_{c(v) \backslash\{i\}}\right)$ (such a $T$ exists, as $v_{c(v) \backslash\{i\}}$ is not the zero game). Suppose $j \notin T$. Then, $T \notin \mathcal{M}_{j}\left(v_{c(v) \backslash\{i\}}\right)=\mathcal{M}_{l}\left(v_{c(v) \backslash\{i\}}\right)$ for any $l \in D_{j}$. In particular, $T \subseteq c(v) \backslash\left(D_{j} \cup\{i, j\}\right) \subseteq E_{j} \backslash\{i\} \subsetneq S$, contradicting the minimality of $S$. Hence, $j \in T$ for all $T \in \mathcal{M}\left(v_{c(v) \backslash\{i\}}\right)$.

This means that $v_{c(v) \backslash\{i, j\}}$ is the zero game and therefore, $i$ is pivotal in $v_{c(v) \backslash\{j\}}$. By the initial condition, $v_{c(v) \backslash\{j\}}$ must be unanimous, and as $S \in \mathcal{M}\left(v_{c(v) \backslash\{j\}}\right)$ it must hold that $\{S\}=\mathcal{M}\left(v_{c}(v) \backslash\{j\}\right) \supseteq \mathcal{M}\left(v_{c(v) \backslash\{k\}}\right)$. Hence, as $v_{c(v) \backslash\{k\}}$ is not the zero game, $\{S\}=\mathcal{M}\left(v_{c}(v) \backslash\{k\}\right)$, in contradiction to $S \in \mathcal{M}_{k}(v)$. Hence, there is no $S \in \mathcal{M}_{k}\left(v_{c}(v) \backslash\{j\}\right)$ and therefore $\mathcal{M}_{k}(v)=\mathcal{M}_{j}(v)$.

2. Let $k \in E_{j}$. I first show that there is $i \in N$ such that $\left|\mathcal{M}_{j}\left(v_{c(v) \backslash\{i\}}\right)\right|=$ $\left|\mathcal{M}_{k}\left(v_{c(v) \backslash\{i\}}\right)\right|$. By the induction hypothesis, it is sufficient to show that there is $i \in N$ such that either both $j$ and $k$ are null players in $v_{c}(v) \backslash\{i\}$ or neither of them is. Equivalently, either $i \in D_{j} \cap D_{k}$ or $i \notin D_{j} \cup D_{k}$.

Suppose that such $i$ does not exist. In this case, $c(v)=D_{j} \cup D_{k}$ and $D_{j} \cap D_{k}=\emptyset$. From the first part of the proof, it follows that $l \in D_{j}$ if and only if $j \in D_{l}$. In 
particular, each $l \in D_{j}$ is a null player in $v_{c(v) \backslash\{j\}}$. Hence, $v\left(c(v) \backslash\left(D_{j} \cup\{j\}\right)\right)=$ $v(c(v) \backslash\{j\})=1$. But this means that $v\left(D_{k}\right)=v\left(c(v) \backslash D_{j}\right)=1$ and for the same reasons $v\left(D_{j}\right)=1$. Hence, as $D_{j}$ and $D_{k}$ are disjoint, $v$ is not proper-a contradiction.

Therefore, there must be $i \in N$ such that $\left|\mathcal{M}_{j}\left(v_{c(v) \backslash\{i\}}\right)\right|=\left|\mathcal{M}_{k}\left(v_{c}(v) \backslash\{i\}\right)\right|$. For such $i$ it holds that

$$
\begin{aligned}
\left|\mathcal{M}\left(v_{c(v) \backslash\{j\}}\right)\right| & =\left|\mathcal{M}\left(v_{c(v) \backslash\{i, j\}}\right)\right|+\left|\mathcal{M}\left(v_{c(v) \backslash\{k, j\}}\right)\right|-\left|\mathcal{M}\left(v_{c(v) \backslash\{i, k, j\}}\right)\right| \\
& =\left|\mathcal{M}\left(v_{c(v) \backslash\{i\}}\right)\right|-\left|\mathcal{M}_{j}\left(v_{c(v) \backslash\{i\}}\right)\right|+\left|\mathcal{M}\left(v_{c}(v) \backslash\{k, j\}\right)\right|-\left|\mathcal{M}\left(v_{c(v) \backslash\{i, k, j\}}\right)\right| \\
& =\left|\mathcal{M}\left(v_{c(v) \backslash\{i\}}\right)\right|-\left|\mathcal{M}_{k}\left(v_{c(v) \backslash\{i\}}\right)\right|+\left|\mathcal{M}\left(v_{c(v) \backslash\{k, j\}}\right)\right|-\left|\mathcal{M}\left(v_{c(v) \backslash\{i, k, j\}}\right)\right| \\
& =\left|\mathcal{M}\left(v_{c(v) \backslash\{i, k\}}\right)\right|+\left|\mathcal{M}\left(v_{c(v) \backslash\{j, k\}}\right)\right|-\left|\mathcal{M}\left(v_{c(v) \backslash\{i, j, k\}}\right)\right| \\
& =\left|\mathcal{M}\left(v_{c(v) \backslash\{k\}}\right)\right| .
\end{aligned}
$$

Consequently,

$$
\begin{aligned}
\left|\mathcal{M}_{j}(v)\right| & =|\mathcal{M}(v)|-\left|\mathcal{M}\left(v_{c}(v) \backslash\{j\}\right)\right| \\
& =|\mathcal{M}(v)|-\left|\mathcal{M}\left(v_{c}(v) \backslash\{k\}\right)\right| \\
& =\left|\mathcal{M}_{k}(v)\right| .
\end{aligned}
$$

I have shown that $\left|\mathcal{M}_{j}(v)\right|=\left|\mathcal{M}_{k}(v)\right|$ for all $j, k \in c(v)$. By the induction hypothesis $\left|\mathcal{M}_{j}\left(v_{S}\right)\right|=\left|\mathcal{M}_{k}\left(v_{S}\right)\right|$ for all subsets $S \subsetneq c(v)$ and all $j, k \in c\left(v_{S}\right)$.

The condition on a simple game given in Theorem 3, namely that in every subgame every non-null player is contained in the same number of minimal winning coalitions, is quite strong; it is satisfied, for instance, by simple games in which all players are symmetric. Nevertheless, the latter is not a necessary condition as can be seen in the simple game with minimal winning coalitions $\{1,2,3\},\{3,4,5\}$, and $\{1,2,4,5\}$.

\subsection{The Shapley-Shubik index}

The Shapley-Shubik index (Shapley 1953; Shapley and Shubik 1954) is defined as

$$
\sigma_{j}(v, N)=\sum_{S \subseteq N, j \in S} \frac{(|N|-|S|) !(|S|-1) !}{|N| !}(v(S)-v(S \backslash\{j\})) .
$$

It is well known that $\sigma$ does not depend on $N$, I will therefore simply write $\sigma(v)$ for $\sigma(v, N)$. Also, $\sigma$ is monotonic (Sagonti 1991) and therefore exhibits the strong paradox of smaller coalitions on all games not satisfying the condition in Theorem 3 . But this condition is, in fact, both necessary and sufficient.

Corollary 3 Let $v$ be a simple game. Then, the Shapley-Shubik index does not exhibit the strong paradox of smaller coalitions on $v$ if and only if $\left|\mathcal{M}_{i}\left(v_{S}\right)\right|=\left|\mathcal{M}_{j}\left(v_{S}\right)\right|$ for all winning coalitions $S$ and all $i, j \in c\left(v_{S}\right)$. 
Proof By be Lemma 2 and Theorem 3, it is necessary that $\left|\mathcal{M}_{i}\left(v_{S}\right)\right|=\left|\mathcal{M}_{j}\left(v_{S}\right)\right|$ for all winning coalitions $S$ and all $i, j \in c\left(v_{S}\right)$. To show that this is sufficient, I show that $\sigma_{j}(v) \leq \max _{T \in \mathcal{M}_{j}} \frac{1}{|T|}$ for any such $v$. In this case, for any coalition $S$, a minimal winning coalition $T \subseteq S$ of minimal cardinality delivers higher power for each player in $T$ than $S$. The claim is shown by induction over $n=|c(v)|$. It is clearly true if $v$ is unanimous, in particular, it is true if $n \leq 2$. Hence, let $n \geq 3$ and let $v$ not be unanimous. Then, $v_{c}(v) \backslash\{k\}$ is not the zero game for any $k$. Let $T$ be a minimal winning coalition of minimal cardinality and let $j \in T$. Then,

$$
\sigma_{j}(v)=\frac{1}{|c(v)|} \sum_{k \neq j} \sigma_{j}\left(v_{c(v) \backslash\{k\}}\right) \leq \frac{|c(v)|-1}{|c(v)|} \frac{1}{|T|} \leq \frac{1}{|T|}
$$

by the induction hypothesis.

\section{Conclusion}

I have shown that some natural properties one would expect from power indices contradict each other on many simple games. Nevertheless, these two properties are reasonable in different contexts: while monotonicity seems very natural for games with the same carrier, non-exhibition of the paradox of new players (or smaller coalitions) usually concerns games with different carriers.

There are authors arguing that the paradox of new members is not paradoxical (Felsenthal and Machover 1995) on the grounds that the power of a player is a global property that involves the whole structure of the game. Although this is clearly true, it makes the (equivalent) paradox of smaller coalitions even more peculiar: a player has less power in a smaller coalition despite the fact that this coalition is part of the same game and the only difference is that (other) players are left out.

It therefore seems that monotonic power indices (including the Shapley index and the Banzhaf index) are not the right instrument to measure power in a council or parliament where the formation of coalitions is likely. It rather makes a case for power measures that take into account players' options both within and outside coalitions, such as the bargaining set (Davis and Maschler 1963), or more recently a class of solutions investigated in Karos (2013) where players' power is based on bargaining solutions.

Acknowledgments I would like to thank Steven Brams for his comments, as well as the referees and the editor in charge for their insightful suggestions.

Open Access This article is distributed under the terms of the Creative Commons Attribution 4.0 International License (http://creativecommons.org/licenses/by/4.0/), which permits unrestricted use, distribution, and reproduction in any medium, provided you give appropriate credit to the original author(s) and the source, provide a link to the Creative Commons license, and indicate if changes were made. 


\section{References}

Banzhaf, J. (1965). Weighted voting doesn't work: a mathematical analysis. Rutgers Law Review, 19, 317-343.

Brams, S. (1975). Game theory and politics. New York: Free Press.

Brams, S., \& Affuso, P. (1976). Power and size: a new paradox. Theory and Decision, 7, 29-56.

Coleman, J. (1971). Control of collectives and the power of a collectivity to act. In A. Liebermann (Ed.), Social choice (pp. 192-225). New York: Gordon and Breach.

Davis, M., \& Maschler, M. (1963). Existence of stable payoff configurations for cooperative games. Bulletin of the American Mathematical Society, 69, 106-108.

Dimitrov, D., \& Haake, C. (2008). A note on the paradox of smaller coalitions. Social Choice and Welfare, $30,571-579$.

Fara, R., Leech, D., \& Salles, M. (2014). Voting power and procedures-essays in honour of Dan Felsenthal and Moshé Machover. New York: Springer.

Felsenthal, D., \& Machover, M. (1995). Postulate and paradoxes of relative voting power-a critical reappraisal. Theory and Decision, 38, 195-229.

Karos, D. (2013). Bargaining and power. fEEM Working Paper No. 63.2013.

Karos, D. (2014). Coalition formation in general apex games under monotonic power indices. Games and Economic Behavior, 87, 239-252.

Kóczy, L. (2009). Measuring voting power: the paradox of new members vs. the null player axiom. In I. Rudas, J. Fodor, \& J. Kacprzyk (Eds.), Towards intelligent engineering and information technology (pp. 67-78). New York: Springer.

Sagonti, E. (1991). On the strong monotonicity of power indices. International Journal of Game Theory, 20, 13-22.

Shapley, L. (1953). A value for $n$-person games. In H. Kuhn \& A. Tucker (Eds.), Contributions to the theory of games (pp. 307-317). Princeton: Princeton University Press.

Shapley, L., \& Shubik, M. (1954). A method for evaluating the distribution of power in a committee system. The American Political Science Review, 48, 787-792.

Shenoy, P. (1979). On coalition formation: a game-theoretical approach. International Journal of Game Theory, 8, 133-164.

Young, H. (1985). Monotonic solutions of cooperative games. International Journal of Game Theory, 14, 65-72. 